\title{
ECC Based Defense Scheme for Selective Drop Attack in Wireless Ad Hoc Network
}

\author{
Sangeetha V \\ PG Scholar- M.E Wireless communication \\ PSG College of Technology \\ Coimbatore, India
}

\author{
Dr. G. Umamaheswari \\ Associate Professor \\ PSG College of Technology \\ Coimbatore, India
}

\begin{abstract}
Performance and security are two critical functions of wireless ad-hoc networks (WANETs). Network security ensures the integrity, availability, and performance of WANETs. It helps to prevent critical service interruptions and increases economic productivity by keeping networks functioning properly. Since there is no centralized network management in WANETs, these networks are susceptible to packet drop attacks. In selective drop attack, the neighbouring nodes are not loyal in forwarding the messages to the next node. It is critical to identify the illegitimate node, which overloads the host node and isolating them from the network is also a complicated task. Resistive to selective drop attack (RSDA) scheme is proposed to provide effective security against selective drop attack. A lightweight RSDA protocol is proposed for detecting malicious nodes in the network under a particular drop attack. The RSDA protocol can be integrated with the many existing routing protocols for WANETs such as AODV and DSR. It accomplishes reliability in routing by disabling the link with the highest weight and authenticate the nodes using the elliptic curve digital signature algorithm. In the proposed methodology, the packet drop rate, throughput and end to end delay are analysed.

Keywords: wireless ad-hoc networks; resistive to selective drop attack; network security; elliptic curve digital signature algorithm; distance vector routing; ad-hoc on demand distance vector routing
\end{abstract}

\section{INTRODUCTION}

Wireless Ad-Hoc Networks (WANETs) decentralized nature makes suitable for different types of applications, where central nodes cannot be trusted on and may progress the scalability of networks linked to wireless networks, through practical and theoretical confines to the overall size of such networks have been recognized. Minimal configuration and quick deployment make ad hoc networks suitable for emergencies in military or natural disasters conflicts. The existence of adaptive and dynamic routing protocol enables ad hoc networks to be formed quickly [1]. The applications can further classify wireless Ad-hoc networks into Vehicular Ad hoc Networks (VANETs), Mobile Ad hoc Networks (MANETs), Smartphone Ad-hoc Networks (SPANs), Wireless mesh networks. Wireless Sensor Networks (WSNs) are emerging as a promising technology because of their wide range of applications in industrial, environmental monitoring, military and civilian domains. Due to economic considerations, the nodes are usually simple and low cost. They are often unattended, however, and are hence likely to suffer from different types of novel attacks. A black hole attack (BLA) is one of the most typical attacks and works as follows. The adversary compromises a node and drops all packets that are routed via this node, resulting in sensitive data being discarded or unable to be forwarded to the sink. Because the network makes decisions depending on the nodes' sensed data, the consequence is that the network will completely fail and, more seriously, make incorrect decisions. Therefore, how to detect and avoid BLA is of great significance for security in WSNs. Wireless networks have many different architectures than that of a typical wired network; a host can broadcast that it has the shortest path towards a destination. By doing this, all traffic will be directed to the host that has been compromised, and the host can drop packets at will [2]. The RSDA protocol can provide resistance to selective drop attacks by thwarting the nodes from getting overloaded. It attains reliability in routing using the reliable factor by disabling the link as defective or by obtaining a new efficient route to the destination. To address the selective drop attack, a reliable factor is chosen by computing the list of link weights. If the sum of the weight of a particular route is high, e.g., it indicates that the low reliability, the attacking node can be identified. Each node maintains its own weight; the obtained weight is added to the route request payload. By computing the reliability rate, malicious nodes can be distinguished from other normal nodes. The performance of RSDA protocol is increased compared to existing approaches by considering the factors such as packet drop rate, jitter and routing overhead

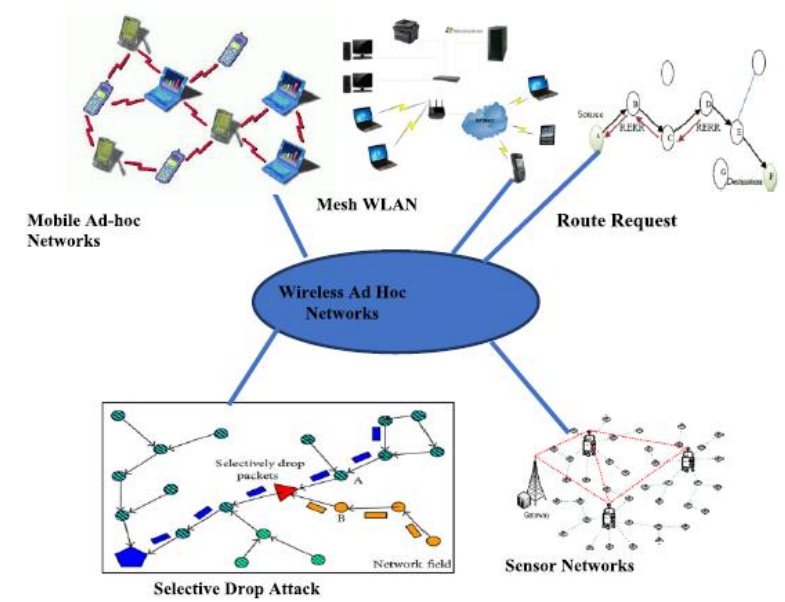

Figure 1. Wireless Ad-Hoc networks (WANETs)

Routing table size is minimized by only including next hop information, not the entire route to a destination node. Sequence numbers for both destination and source are used. Managing the sequence number is the key to efficient routing and route maintenance. Sequence numbers are used to indicate the relative freshness of routing information. Updated by an originating node, e.g., at initiation of route discovery or a route reply. In figure 1 the wireless ad hoc network (WANETS) is a type of local area network (LAN) that is built spontaneously to enable two or more wireless devices to be 
connected to each other without requiring a central device, such as a router or access point. When Wi-Fi networks are in ad-hoc mode, each device in the network forwards data to the others [3]. The RSDA protocol has been designed to offer resistance to selective drop attacks by preventing the nodes from getting overloaded. It achieves reliability in routing using the reliable factor by disabling the link as defective or by obtaining a new efficient route to the destination. This mainly contributes on Wireless Ad Hoc Networks and their security related issues. A review on various protocols is done to deal with selective drop attack in WANET.A light weight RSDA protocol has been proposed for detecting malicious nodes in the network under selective drop attack. The RSDA protocol can be integrated with the many existing routing protocols for WANET such as AODV and DSR. An efficient cryptographic technique ECDSA has been chosen for providing authentication which has a lesser key size however it provides similar security. Finally, it achieves extreme network security measures ensures the integrity, availability, and performance enhanced using RSDA for WANET. In selective drop attack, the malicious nodes would refuse of forwarding message passing through them. At last this attack can potentially drop the throughput of a host to a minimum level. The RSDA protocol has been proposed to strengthen the resistance to selective drop attacks by thwarting the nodes from getting overloaded [4]. It attains reliability in routing by disabling the link as defective or attempts to obtain a new efficient route to the destination.

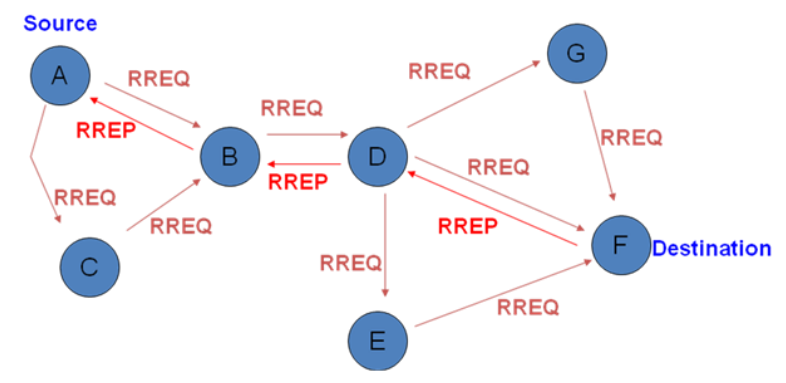

Figure 2. Message routing in AODV

In Figure 2 Route Requests (RREQs), Route Replies (RREPs), and Route Errors (RERRs) are the message types defined by AODV. These message types are received via UDP, and normal IP header processing applies. So, for instance, the requesting node is expected to use its IP address for the message.

\section{PROBLEM STATEMENT}

The packet drop attack can frequently be used to attack WANETs. Wireless networks have many different architectures than that of a typical wired network; a host can broadcast that it has the shortest path towards a destination. By doing this, all traffic will be directed to the host that has been compromised, and the host can drop packets at will. Also over a mobile ad-hoc network, hosts are especially vulnerable to collaborative attacks where multiple hosts will become compromised and deceive the other host on the network. This kind of attacks can be detected at an early stage by monitoring few hundreds of packets based on the number of requests that a host receives from a particular host from a fixed interval of time changes. The early detection of DoS attack prevents the controller going down. If detection happens at early stage then, the impact of flooding of malicious packets can be controlled significantly. The early detection mechanism must be of light weight and should have a high response time [5]. The high response time saves the controller in the period of attack to regain the control by terminating the DoS attack.

\subsection{Software tools used}

Even though there are various tools available to implement the networking technology, the open source software is preferred for its flexible approach towards creating custom algorithms. One such software available to implement the Wireless sensor network test bed is NS2. Network simulation is a technique where a program models the behavior of a network either by calculating the interaction between the different network entities (hosts/routers, data links, packets, etc.) using mathematical formulas, or actually capturing and playing back observations from a production network. When a simulation program is used in conjunction with live applications and services in order to observe endto-end performance to the user desktop, this technique is also referred to as network emulation. Most network simulators use discrete event simulation, in which a list of pending "events" is stored, and those events are processed in order, with some events triggering future events such as the event of the arrival of a packet at one node triggering the event of the arrival of that packet at a downstream node.

\section{IMPLMENTATION}

Soft security mechanism was proposed as a fully distributed trust-based public key management technique for MANET. A Composite Trust-based Public Key Management (CTPKM) was proposed to maximize the performance by mitigating the vulnerabilities. A trusted threshold was fixed with each node to decide whether to trust another node or not. A mechanism based on the simple rate-based control packet forwarding mechanism to alleviate malicious control packet was proposed. It was made secure against other DDoS attacks, and those legitimate nodes are not erroneously treated as misbehavior node. Anti Black Hole (ABM) mechanism, which estimates the suspicious value of a node according to the amount of abnormal difference between RREQs and RREPs transmitted from the node [6]. Multipath forwarding technique to identify attacks in a wireless sensor network based on selective forwarding attack procedure was proposed. Thus it is necessary to propose a strong security based system for IoT based wireless sensor network. Our proposed work handles this effectively. To address the selective drop attack, a reliable factor is chosen by computing the list of link weights. If the sum of the weight of a particular route is high, e.g., it indicates that the low reliability, the attacking node can be identified. Each node maintains its own weight; the obtained weight is added to the route request payload. By computing the reliability rate, malicious nodes can be distinguished from other normal nodes. Unicast delivers a message to a single specified node; Broadcast delivers a message to all nodes in the network; Multicast delivers a message to a group of nodes that have expressed interest in receiving the message; Anycast delivers a message to any one out of a group of nodes, typically the one nearest to the source. RSDA provides an effective security for selective drop attack. It attains reliability in routing using the reliable factor by disabling the link as defective or by obtaining a new efficient route to the destination [7].

In the below figure 3 represents architecture of proposed system, in which all modules of the work are represented. Wireless network initialized and nodes are assigned with AES and ECC key and verified on message transfer. The design is a plan or drawing produced to show the look and 
function or workings of an object before it is made. Unified Modeling language (UML) is a standardized modeling language enabling developers to specify, visualize, construct and document artifacts of a software system. Thus, UML makes these artifacts scalable, secure and robust in execution. UML is an important aspect involved in objectoriented software development. It uses graphic notation to create visual models of software systems.

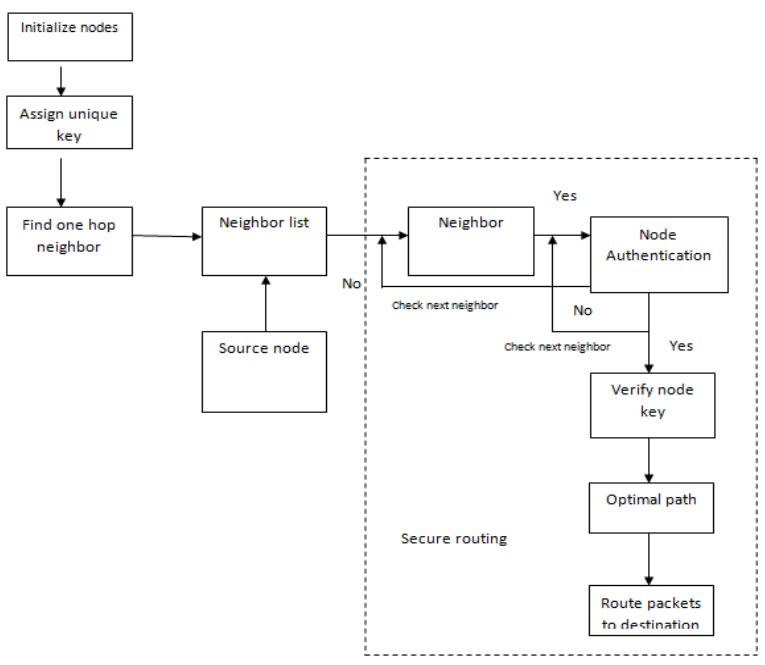

Figure 3. Architecture diagram

Wireshark is a packet sniffer and analysis tool. It captures network traffic on the local network and stores that data for offline analysis. Wireshark captures network traffic from Ethernet, Bluetooth, Wireless (IEEE.802.11), Token Ring, Frame Relay connections, and more. Wireshark intercepts traffic and converts that binary traffic into human-readable format. This makes it easy to identify the type of traffic is crossing the network. In Figure 4 it shows the analyzing of duplicate packets using Wireshark.

\begin{tabular}{|c|c|c|c|c|c|}
\hline 10. & The & sarce & Destratan & \multicolumn{2}{|c|}{ Arotad legen into } \\
\hline & 311.599800133 & de:ef:2d:af:3e:5a & & & 4410.0 .0 .3 is at de:of:2d:af:3e:5a \\
\hline & 321.539095555 & de:2f:2d:af:3et:5a & & ARP & 410.0 .0 .3 is at de:af:20d:af:3e:5a \\
\hline & 331.585651357 & de:Of:2d:af:3e:5a & & $A R P$ & 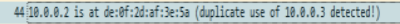 \\
\hline & 341.555575991 & de:af:2d:af:3e:5a & & ARP & 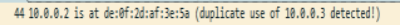 \\
\hline & 351.565577991 & de:0f:2d:af:3e:5a & & ARP & 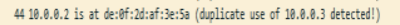 \\
\hline & 361.565581397 & de:Pf:2d:af:3e:5a & & ARP & 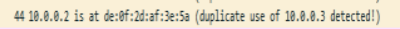 \\
\hline & 372.4083231455 & 18.9.8.2 & 10.8.8.3 & IOPP & 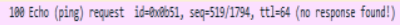 \\
\hline & 382.2 .45244233 & 19.9.8.2 & 18.8.8.3 & IOP & 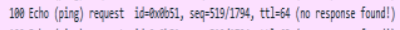 \\
\hline & 392.408585477 & 10.9.8.2 & 18.9.8.3 & IOP & 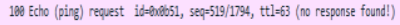 \\
\hline & 402.448621758 & 10.9.8.2 & 10.8.8.3 & IOP & 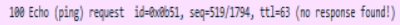 \\
\hline & & & 10002 & & 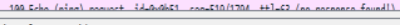 \\
\hline \multicolumn{6}{|c|}{ :33: 4 bytes on nire $(352$ bits), 4} \\
\hline \multicolumn{6}{|c|}{$\begin{array}{l}\text { Linux cooked capture } \\
\text { address Resolution Protocol }\end{array}$} \\
\hline \\
\hline \multicolumn{6}{|c|}{ 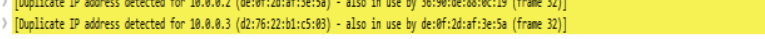 } \\
\hline
\end{tabular}

Figure 4. Analyzing duplicate packets using Wireshark

Since attacker replicates his own MAC address between target and client, the ARP table gets updated with replicated MAC address. Each switch has an ARP (Address Resolution Protocol) table in order to store the IP addresses and MAC addresses of the network devices. The ARP table is used to determine the destination MAC addresses of the network nodes. Thus by analyzing packets after ARP spoofing, the MAC address gets replicated between the hosts with the different IP address in ARP table. Here, the detection is based on IP-MAC addresses bindings. Once the topology gets initialized, the controller will dynamically allocates MAC address for every IP address in the network. The controller will store that created MAC address in MAC_to_port table. During attack, the attacker duplicates his own MAC address and spoofs arrived packets. But the controller doesn't knows whether arrived packets having legimate MAC address or duplicated MAC address [8].

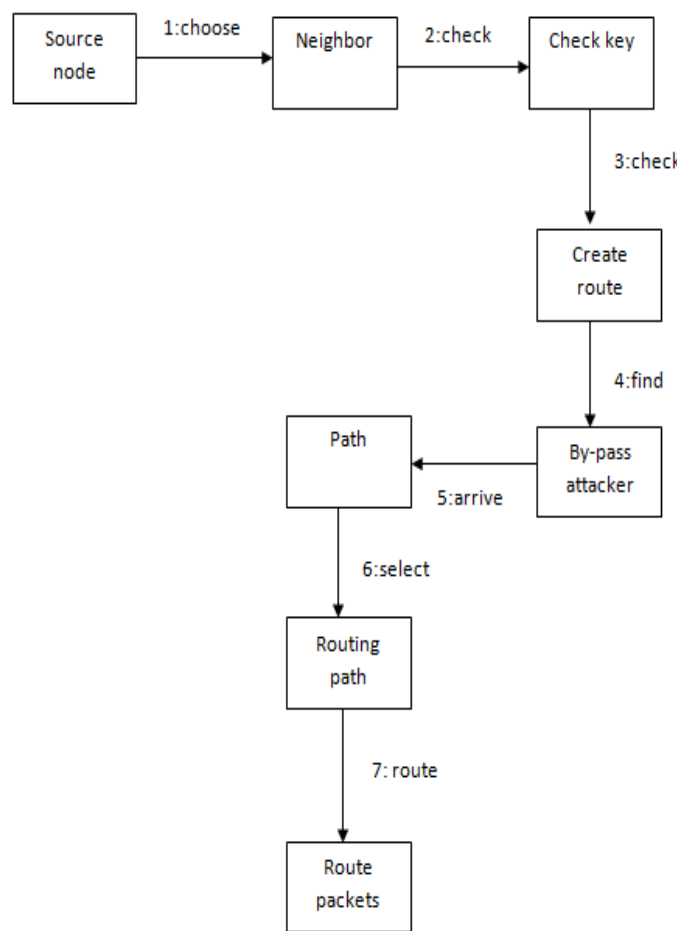

Figure 5. Process flow

The above Figure 5 represents collaboration diagram, it represent the process flow and its function. Assigning trust parameter, calculating fitness function and route selection are the sequence of process handled. It clear cut the process involved in the proposed work were the source node choose the neighbor node and check the key and it creates the routes path for sending the packets and also find the By-pass attacker which arrive in the path. thus the algorithms involved will avoid the attackers in the path and find the best routing path for allowing the route packets. Minimal configuration and quick deployment make ad hoc networks suitable for emergencies in military or natural disasters conflicts. The applications can further classify wireless Ad-hoc networks into Vehicular Ad hoc Networks, Mobile Ad hoc Networks, Smartphone Ad-hoc Networks, Wireless mesh networks and so on. 


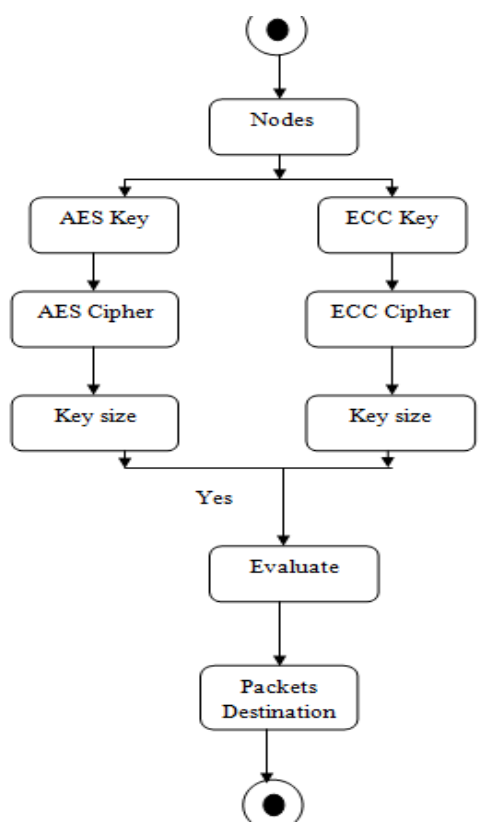

Figure 6. Activity diagram

The above Figure 6 show the activity diagram of the proposed system, where we represented the identified activities and its functional flow. The selected node will check the AES key and ECC key. At the next step it check for AES cipher and ECC cipher for evaluating the key size and send the packets to the destinations [9]. The modules included in our implementation are as follows Network model, AES implementation, ECC implementation, Key size Evaluation.

\subsection{Network model}

Consider a wireless sensor network consisting of sensor nodes that are uniformly and randomly scattered in a circular network; the network radius is $\mathrm{R}$, with nodal density $\mathrm{p}$, and nodes do not move after being deployed. Upon detection of an event, a sensor node will generate messages, and those messages must be sent to the sink node. Consider that linklevel security has been established through a common cryptography-based protocol. Thus, consider a link key to be safe unless the adversary physically compromises either side of the link [10].

\subsection{AES implementation}

AES performs all its computations on bytes rather than bits. Hence, AES treats the 128 bits of a plaintext block as 16 bytes. These 16 bytes are arranged in four columns and four the third mixes columns. The last transformation is a simple exclusive or (XOR) operation performed on each column using a different part of the encryption key longer keys need more rounds to complete. To decrypt an AES-encrypted ciphertext, it is necessary to undo each stage of the encryption operation in the reverse order in which it is applied [11].

\subsection{ECC implementation}

Elliptical curve cryptography (ECC) is a public key encryption technique based on elliptic curve theory that can be used to create faster, smaller, and more efficient cryptographic keys. ECC generates keys through the properties of the elliptic curve equation instead of the traditional method of generation as the product of very large prime numbers. Because ECC helps to establish equivalent security with lower computing power and battery resource usage.

\subsection{Key generation}

Key generation is an important part where an algorithm should generate both public key and private key. The sender will be encrypting the message with receiver's public key and the receiver will decrypt its private key. Now, select a number, $d$ within the range of $n$. Generate the public key using the following equation,

$$
\mathrm{Q}=\mathrm{d} * \mathrm{P}
$$

Where $\mathrm{d}=$ the random number selected within the range of (1 to $\mathrm{n}-1)$. $\mathrm{P}$ is the point on the curve, $\mathrm{Q}$ is the public key and $\mathrm{d}$ is the private key.

\subsection{Encryption}

Let ' $M$ ' be the message that has to be sent. Consider ' $M$ ' has the point ' $\mathrm{P}$ ' on the curve ' $\mathrm{E}$ '. Randomly select ' $\mathrm{k}$ ' from [1 (n-1)]. Cipher texts will be generated let C.

$$
\mathrm{C}=\mathrm{M}+(\mathrm{k} * \mathrm{P})
$$

\subsection{Decryption}

Use the following equation to get back the original message ' $M$ ' that was sent.

$$
\mathrm{M}=\mathrm{C}-\mathrm{d} *(\mathrm{k} * \mathrm{P})
$$

$\mathrm{M}$ is the original message that was sent.

\subsection{Key size implementation}

The key are uniquely generated and assigned for all nodes in the network and used for encryption. The cipher text generated with the key. The keys generated for nodes using AES and ECC are stored in a text files. The size of key are compared. ECC's main advantage is that you can use smaller keys for the same level of security, especially at high levels of security. The implementation shows that the Key size of ECC is very small compared to AES.

\section{RESULTS AND DISSCUSION}

Cryptographic schemes employ large keys and many rounds in their design for protection against malicious attacks. Wireless ad-hoc network thus requires adequate securityensuring approaches that consume less power and show high performance. However, providing these requirements in one design is an impossible task because. Thus, developing a lightweight cryptographic algorithm is critical because the cryptographer must address security-performance, securitycost, performance-cost. In the proposed system, we used highly secure algorithm and also we can by-pass the attacker node. After finishing the development of any computer based system the next complicated time consuming process is system testing. During the time of testing only the development company can know that, how far the user requirements have been met out, and so on. Software testing is an important element of the software quality assurance and represents the ultimate review of specification, design and coding. The increasing feasibility of software as a system and the cost associated with the software failures are motivated forces for well-planned through testing.

- $\quad$ Source code testing

- Specification testing

- Module level testing

- Unit testing

- Integration testing

- Validation testing

- Performance testing

- $\quad$ Black box testing 
- Output testing

- User acceptance testing

Table 1. Unit testing - test cases

\begin{tabular}{|l|l|l|l|l|l|}
\hline $\begin{array}{l}\text { S. } \\
\text { O }\end{array}$ & Test & Test & Test \\
Description & Procedure & $\begin{array}{l}\text { Expected } \\
\text { Result }\end{array}$ & $\begin{array}{l}\text { Actual } \\
\text { Result }\end{array}$ \\
\hline 1 & $\begin{array}{l}\text { To check } \\
\text { routing table }\end{array}$ & $\begin{array}{l}\text { Input nodes } \\
\text { ids }\end{array}$ & $\begin{array}{l}\text { Execute } \\
\text { robust.tc } \\
1\end{array}$ & $\begin{array}{l}\text { Nodes are } \\
\text { created } \\
\text { and } \\
\text { deployed }\end{array}$ & $\begin{array}{l}\text { Routing } \\
\text { table is } \\
\text { created }\end{array}$ \\
\hline 2 & $\begin{array}{l}\text { To check path } \\
\text { creation }\end{array}$ & $\begin{array}{l}\text { Input source } \\
\text { and } \\
\text { destination }\end{array}$ & $\begin{array}{l}\text { Execute } \\
\text { robust.tc } \\
1\end{array}$ & $\begin{array}{l}\text { Routes are } \\
\text { created }\end{array}$ & $\begin{array}{l}\text { Routing } \\
\text { table is } \\
\text { created }\end{array}$ \\
\hline 3 & $\begin{array}{l}\text { To check } \\
\text { authentication }\end{array}$ & $\begin{array}{l}\text { Snput Key of } \\
\text { Source node }\end{array}$ & $\begin{array}{l}\text { Execute } \\
\text { robust.tc } \\
1\end{array}$ & $\begin{array}{l}\text { Node Key } \\
\text { values are } \\
\text { checked }\end{array}$ & $\begin{array}{l}\text { Node is } \\
\text { authenti } \\
\text { c with } \\
\text { true flag }\end{array}$ \\
\hline 4 & $\begin{array}{l}\text { To check } \\
\text { packet delivery }\end{array}$ & $\begin{array}{l}\text { Input Packets } \\
\text { from Source } \\
\text { node }\end{array}$ & $\begin{array}{l}\text { Execute } \\
\text { robust.tc } \\
1\end{array}$ & $\begin{array}{l}\text { Nodes } \\
\text { should get } \\
\text { packets } \\
\text { delivered }\end{array}$ & $\begin{array}{l}\text { Nodes } \\
\text { received } \\
\text { packets } \\
\text { and } \\
\text { monitor } \\
\text { ed by } \\
\text { sink for } \\
\text { packet } \\
\text { evaluati } \\
\text { ons }\end{array}$ \\
\hline
\end{tabular}

Table 2: System specification

\begin{tabular}{l|l}
\hline Parameters & Values \\
\hline Processor & Intel(R) Core(TM) i3 -32227U \\
& CPU @ 1.90GHz \\
\hline RAM & 4 GB \\
\hline Diskspace & 255GB \\
\hline Operating system & Windows 10 \\
\hline Application Software & Win2000/XP / Linux 9.0 \\
& VM ware Workstation \\
\hline
\end{tabular}

The simulation is carried out in ns-aallinone-2.34 with 33 number of nodes initialized in wireless network and routing is done is AODV protocol. The nam animator size is set at 1000 X1000 and neighbor nodes are detected with the distance $<250$. Nodes are assigned with AES and ECC keys for secure network.

The comparison of key size between AES algorithm and ECC algorithm, and it clearly indicates that the key size of ECC algorithm is very less compared to AES key size. The network communication cost can be reduced with ECC implementation. The key are uniquely generated and assigned for all nodes in the network and used for encryption. The cipher text generated with the key. The keys generated for nodes using AES and ECC are stored in a text files. The size of key are compared. ECC's main advantage is that you can use smaller keys for the same level of security, especially at high levels of security. The implementation shows that the Key size of ECC is very small compared to AES.

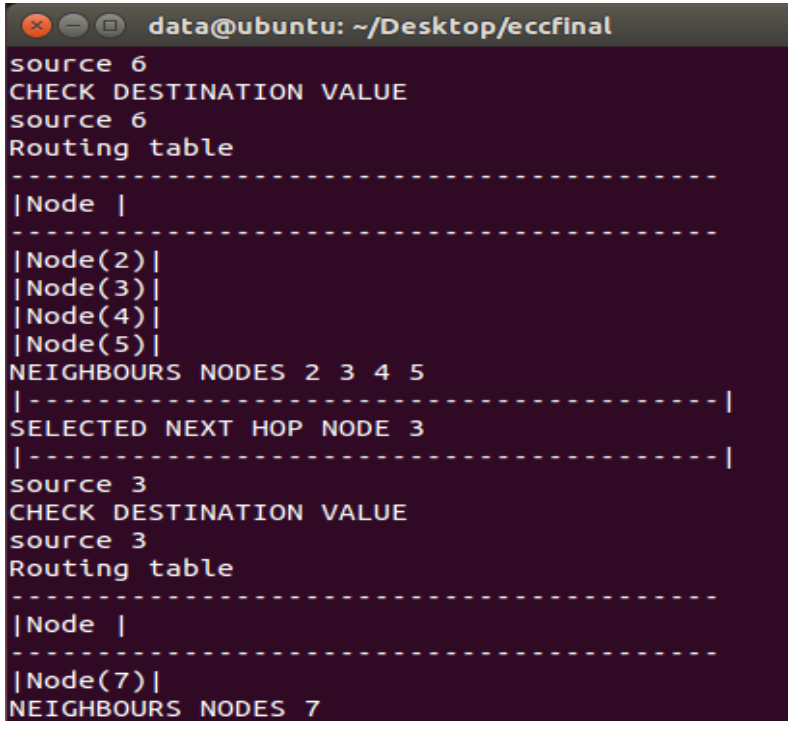

Figure 7. Routing path creation

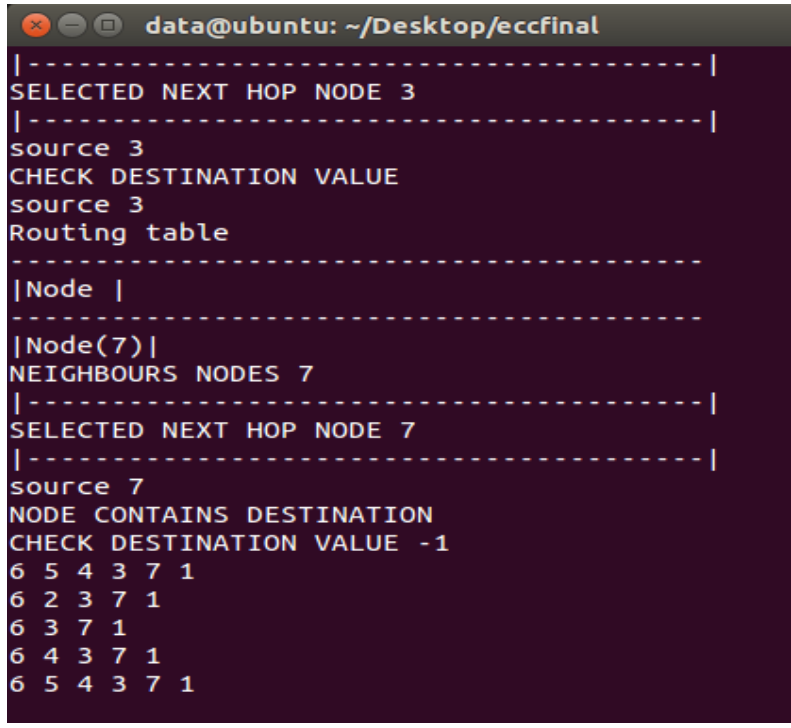

Figure 8. List of available paths

The Figure 7 and 8 shows the routing path and list of available paths which is based on the neighboring node detection table and it finds the best path and next hop node. The selected source node contains destination and check the destination values. In RSDA, AREQ is appended with the RREQ packet to accumulate the transmission rate value. The selective drop attack can be identified by checking the difference of a node with 2-hop neighbors and the nodes threshold value. The keys generated for nodes using AES and ECC are stored in a text files. The size of key are compared. ECC's main advantage is that you can use smaller keys for the same level of security, especially at high levels of security. The node notices an extreme variance, then the link will be disjointed from its parent as defective or malicious and takes up the responsibility for searching a new route to the destination. 


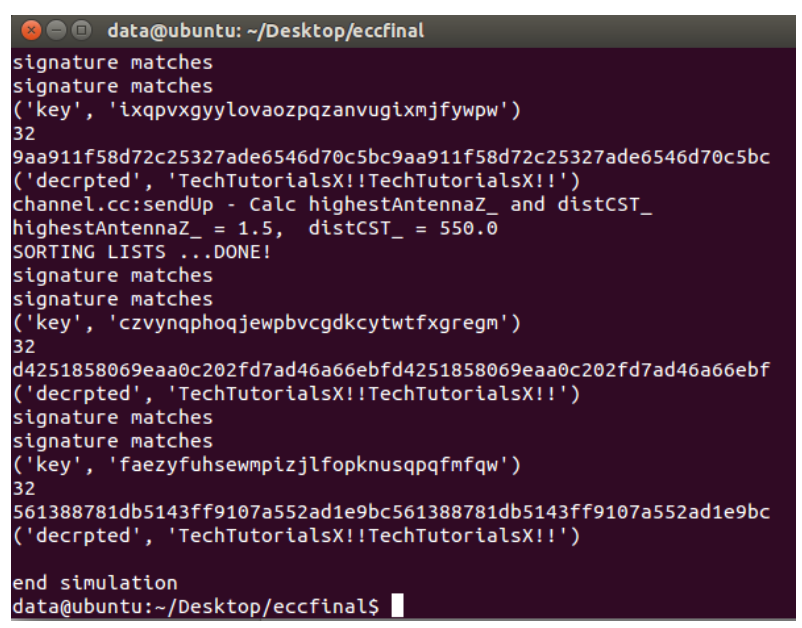

Figure 9. ECC and AES signature checking in the message transmission

In Figure 9 the ECC and AES signature checking is done in message transmission were the key size is compared and then the signature should matches the key size and avoid the selective drop attack during the transmission of the message to the receiver. Thus the simulation process end by delivering the packets without any loss in the packets due to selective drop in the packet transmission.

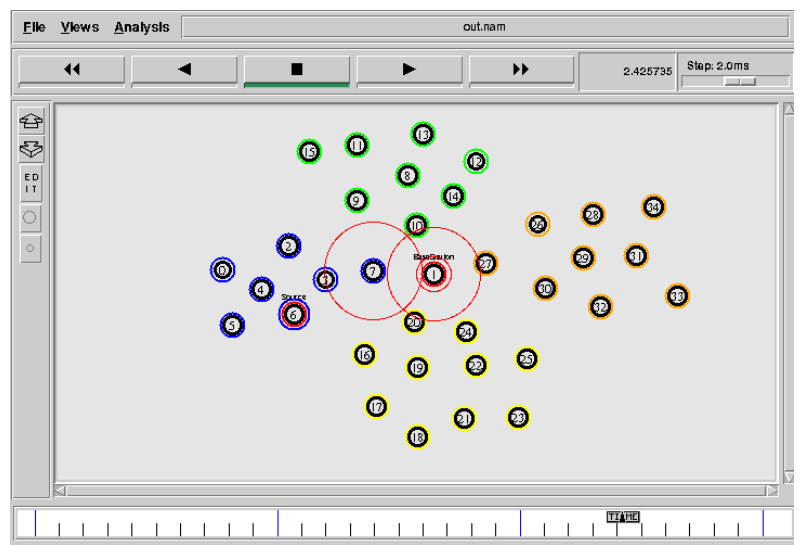

Figure 10. packet sending in nam animator

The network throughput, packet loss and end to end delay are registered and plotted below. The below graph defines the delay in the simulation phase. $\mathrm{X}$ axis time in (seconds) and $\mathrm{Y}$ axis delay time in (milliseconds). The experiment was running 5 seconds of time. End to End Delay refers to the time taken for a packet to be transmitted across a network from source to destination during the simulation time.

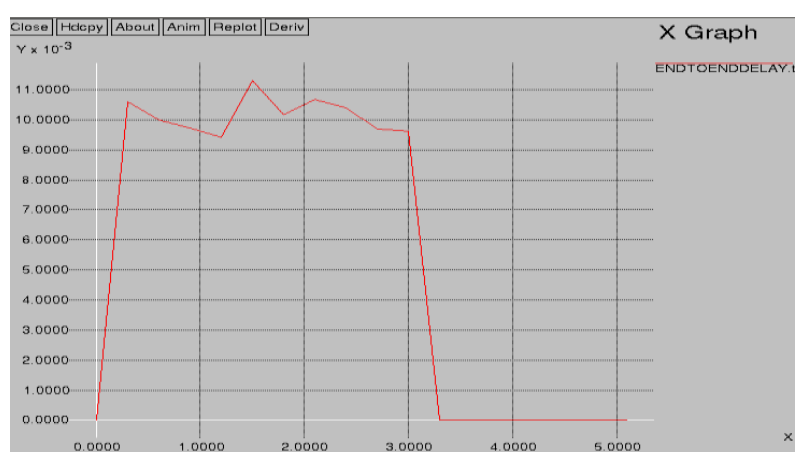

Figure 11. End to End Delay analysis
End-to-end encryption is a system of communication where only the communicating users can read the messages. It is necessary to calculate the execution time in order to evaluate the efficiency of the algorithm. The specific feature is communicating with the each nodes of the network rather than communicating with the intermediary node that exist to establish the network.

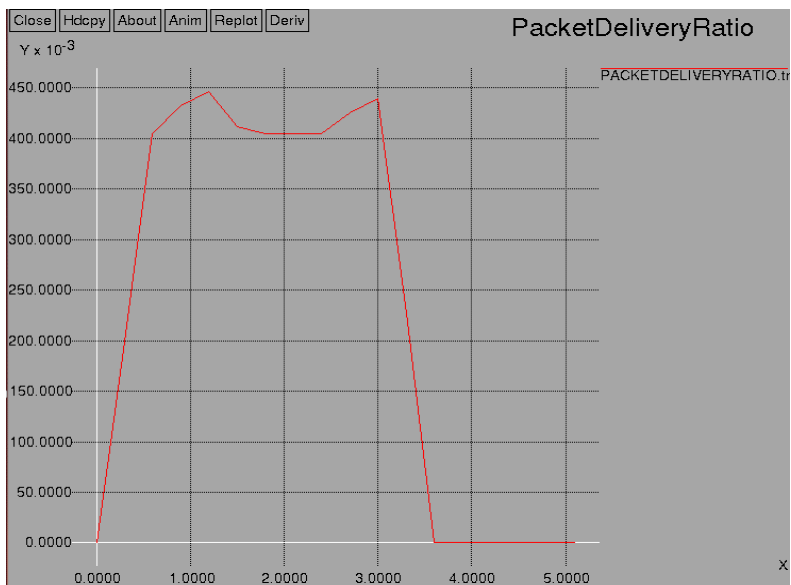

Figure 12. Throughput Analysis

The above graph defines the throughput for the proposed protocol. $\mathrm{X}$ axis time in (seconds) and $\mathrm{Y}$ axis throughput (bit/s) The experiment was running 5 seconds of time. Throughput is the rate at which a network sends receives data. It is a good channel capacity of net connections and rated in terms bits per second (bit/s). Throughput is the rate of successful message delivery over a communication channel by using the algorithms and to resist the selective drop attack in wireless ad hoc network.

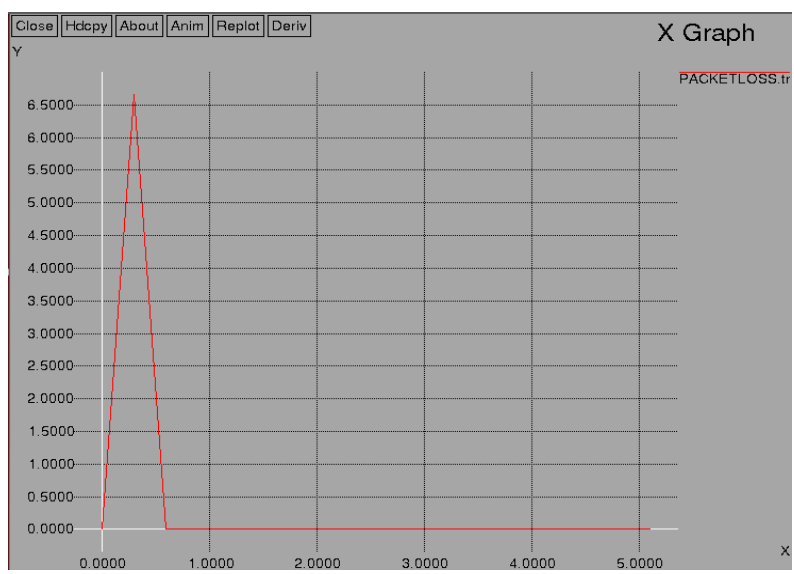

Figure 13. Packet loss analysis

The above graph defines the packet loss for the proposed protocol. $\mathrm{X}$ axis time in (seconds) and $\mathrm{Y}$ axis shows packet loss. The experiment was running 5 seconds of time. Packet loss is the number of packets lost at which a network sends receives data. Packet loss is responsible for many of the network issues especially in WAN connectivity and WiFi networks. The result from the above graph is that reason behind it like the issue is due to network connectivity or the quality of the network degrades due to TCP or UDP packet loss. This packet loss may happen if a router is receiving more data than it transmit or it forgot packets. 
Table 3: Simulation parameter

\begin{tabular}{|l|l|}
\hline Parameter & Values \\
\hline Simulator & ns-2 \\
\hline Simulation Area & $1000 \times 1000 \mathrm{~m}$ \\
\hline Mobile Nodes & 33 \\
\hline Node Speed & $20 \mathrm{~m} / \mathrm{s}$ \\
\hline Packet Size & 64 byte \\
\hline Rate of Transmission of Packets & 4 Packets per Second \\
\hline Routing Protocols & AODV \\
\hline Security Algorithms & AES, ECC \\
\hline Simulation Time & 5 seconds \\
\hline
\end{tabular}

The reserved bits in RREQ and RREP packets are used for analyzing the total number of packets sent by the source node and the destination node verifies the same. AREQ and Authenticated Route Reply (AREP) packets are used for checking the transmission rate. ECDSA based authentication code is attached with the message to mitigate from tampering of messages. In RSDA, AREQ is appended with the RREQ packet to accumulate the transmission rate value. The selective drop attack can be identified by checking the difference of a node with 2-hop neighbors and the nodes threshold value.

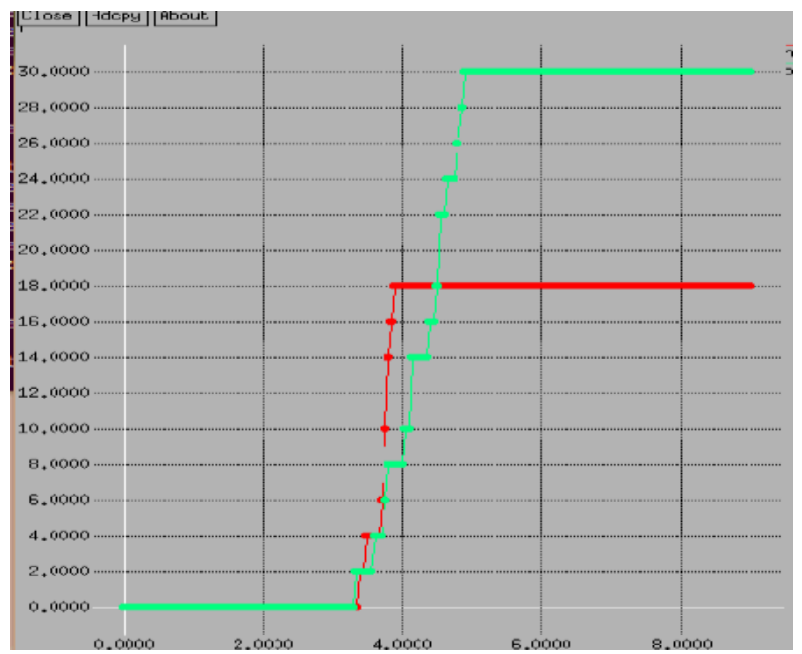

Figure 14. comparison of packet loss with and without RSDA

If the transmission rate is increased or decreased extensively, then the data transmission advances towards the destination, each node checks the weight of the link. If the variation between the last rate in AREQ and the node's estimated value is greater than the threshold value, then it is proven that at least one malicious node exists between nodes and the node is the one which added its last rate to AREQ. If the node notices an extreme variance, then the link will be disjointed from its parent as defective or malicious and takes up the responsibility for searching a new route to the destination.

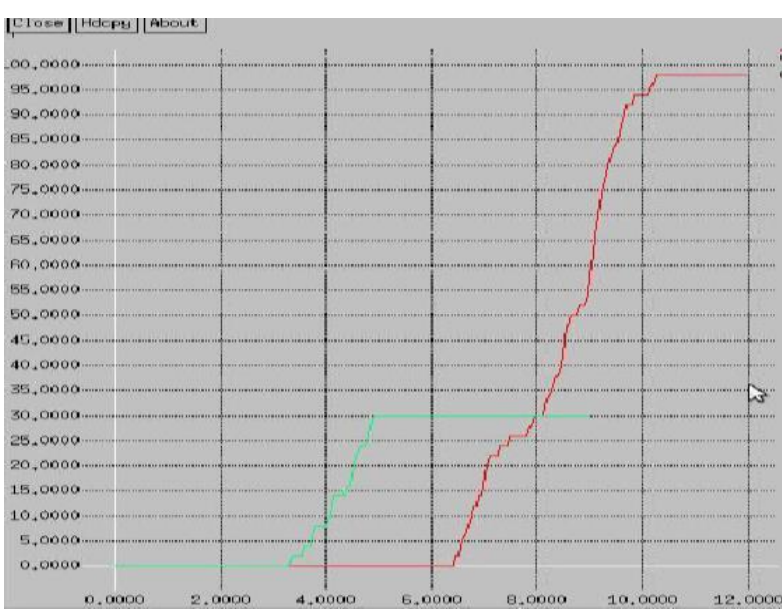

Figure 15. Comparison of Throughput with and without RSDA

The above graphs shows the comparison of Packet loss and Throughput based on AODV existing and proposed algorithms, $\mathrm{X}$ axis shows the time in seconds and $\mathrm{Y}$ axis shows the packet loss and throughput. that shows the result of with and without Selective drop attack. Fig 15 shows that packet loss is decreased without attack and increased with attack in AODV using ECC algorithm. Red line shows that packet loss is reduced with RSDA and green line indicates that packet loss is increased without RSDA.

In Figure 15 The network throughput is more without attack and network throughput is reduced due to selective packet drop attack in the network which is triggered by the malicious node. Red line indicates that with RSDA throughput is increased and green line indicates the reduced throughput without RSDA.

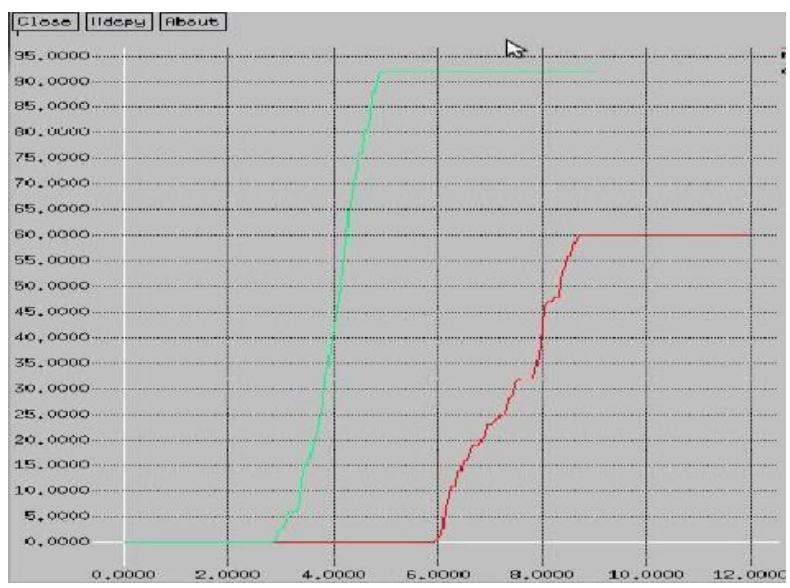

\section{Figure 16. Comparison of end to end delay with and} without RSDA

The above graph shows that end to end delay is more with attack and reduced without attack using AODV protocol in ECC algorithm. Thus the comparison is done based on with and without Resistive selective drop attack in wireless ad hoc network using AODV protocol by proposing the ECC algorithm. 
Table 4: Comparison parameters of with and without RSDA

\begin{tabular}{|l|l|l|l|}
\hline Sr. No. & Parameters & $\begin{array}{l}\text { Without } \\
\text { RSDA }\end{array}$ & With RDSA \\
\hline 1 & $\begin{array}{l}\text { End to End } \\
\text { Delay }\end{array}$ & $92 \mathrm{~ms}$ & $60 \mathrm{~ms}$ \\
\hline 2 & Packet Loss & 30 & 18 \\
\hline 3 & Throughput & 30 bits/s & 95 bits/s \\
\hline
\end{tabular}

There is increased through put, reduced delay of packets and packet loss during the Selective Packet Forward Attack. It can be said that the proposed technique is better as compared to the existing technique. There are various techniques to isolate and prevent selective packet drop attack which degrade the system performance by decreasing throughput, increasing latency and end-to-end delay. In proposed technique delay, packet loss and throughput are better.

\section{CONCLUSION AND FUTURE WORK}

Novelty in the proposed methodology is implementation of elliptical curve cryptography. ECC key is well suited for wireless applications with smaller key size providing secure robust protection for wireless Ad-hoc network. Highly secure algorithm like AES and ECC is used and also can by-pass the attacker node. Using Wireshark the duplicate packets are analyzed and attack detection is identified. The network throughput, packet loss and end to end delay are analyzed and performance Comparison of with and without RSDA is analyzed. Proposed methodology shows improvement in reduction of selective drop attack. Minimal configuration and quick deployment make ad hoc networks suitable for emergencies in military or natural disasters conflicts. The applications can further classify wireless Ad-hoc networks into Vehicular Ad hoc Networks, Mobile Ad hoc Networks, Smartphone Ad-hoc Networks, and Wireless mesh networks.

In proposed work, the keys for encryption are generated using AES algorithm and ECC algorithm. Thus using AODV in wireless Ad-hoc network packet loss, throughput, end to end delay is analyzed. Comparison of with and without Resistive selective drop attack is analyzed. In future, the work can be extended to implementing few more key generation algorithm.

\section{REFERENCES}

[1] R. N. Ode, D. Perdana, and R. F. Sari, "Performance evaluation of AODV, AODV-UU, and AODV with malicious attack mode on vehicular ad-hoc network," Adv. Sci. Lett., vol. 23, no. 5, pp. 3990-3994, 2019.

[2] A. Ranjan, V. Kuthadi, T. Marwala, and R. Selvaraj, "Swarm based archi- tecture for defense against stealthy attacks in mobile ad hoc network," Ad Hoc Sensor Wireless Netw., vol. 36, nos. 1-4, pp. 107-126, 2020

[3] Z. J. Haas, J. Deng, B. Liang, P. Papadimitratos, and S. Sajama, "Wireless ad hoc networks," Encycl. Telecommun., vol. 1, no. 1, pp. 1-28, Dec. 2018.

[4] S. Youse, M. S. Mousavi, and M. Fathy, "Vehicular ad hoc networks (VANETs): Challenges and perspectives," in Proc. 6th Int. Conf. Telecom-mun., 2006, pp. 761-766.
[5] H. Deng, W. Li, and D. P. Agrawal, "Routing security in wireless ad hoc networks," IEEE Commun. Mag., vol. 40, no. 10, pp. 70-75, Oct. 2019.

[6] I. F. Akyildiz, X. Wang, and W. Wang, "Wireless mesh networks: A survey," Comput. Netw., vol. 47, no. 4, pp. 445-487, 2017.

[7] V. Balakrishnan and V. Varadharajan, "Packet drop attack: A serious threat to operational mobile ad hoc networks," in Proc. Int. Conf. Netw. Commun. Syst. (NCS), Krabi, Thailand, 2016, pp. 89-95.

[8] M. Peng,W. Shi, J.-P. Corriveau, R. Pazzi, andY.Wang, "Black hole search in computer networks: State-of-theart, challenges and future directions," J. Parallel Distrib. Comput., vol. 88, pp. 1-15, Feb. 2016.

[9] J.-M. Chang, P.-C. Tsou, I. Woungang, H.-C. Chao, and C.-F. Lai, "Defending against collaborative attacks by malicious nodes in MANETs: A cooperative bait detection approach," IEEE Syst. J., vol. 9, no. 1, pp. 6575, Mar. 2015.

[10] A. Aijaz and A. H. Aghvami, "Cognitive machine-tomachine communications for Internet-of-Things: A protocol stack perspective," IEEE Inter-net Things J., vol. 2, no. 2, pp. 103-112, Apr. 2015.

[11] P. Chen, S. Cheng, and K. Chen, "Information fusion to defend intentional attack in Internet of Things," IEEE Internet Things J., vol. 1, no. 4, pp. 337-348, Aug. 2014.

[12] X. Meng and T. Chen, "Event-driven communication for sampled-data control systems," in Proc. Amer. Control Conf. (ACC), vol. 1, 2013, pp. 3002-3007.

[13] Changhoon Yoon, Seungsoo Lee, Heedo Kang, Taejune Park, 'FloodGuard: A DoS Attack Prevention Extension in Software-Defined Networks', IEEE/ACM transactions on networking, VOL. 25, NO. 6,DEC 2017.

[14] A. K. Khare, J. L. Rana, and R. C. Jain, "Detection of wormhole, blackhole and DDOS attack in MANET using trust estimation under fuzzy logic methodology," Int. J. Comput. Netw. Inf. Secur., vol. 9, no. 7, p. 29, 2017.

[15] C. Karlof and D. Wagner, "Secure routing in wireless sensor networks: Attacks and countermeasures," Ad hoc Netw., vol. 1, no. 2, pp. 293-315, 2018.

[16] A. Nadeem and M. P. Howarth, "An intrusion detection \& adaptive response mechanism for MANETs," Ad Hoc Netw., vol. 13, pp. 368-380, Feb. 2014.

[17] D. Johnson, A. Menezes, and S. Vanstone, "The elliptic curve digital signature algorithm (ECDSA)," Int. J. Inf. Secur., vol. 1, no. 1, pp. 36-63, Aug. 2019.

[18] D. Zhong, H. Lv, J. Han, and Q. Wei, "A practical application combining wireless sensor networks and Internet of Things: Safety management system for tower crane groups,' Sensors, vol. 14, no. 8, pp. 13794-13814, 2016.

[19] C. Perkins, E. Belding-Royer, and S. Das, Ad Hoc onDemand Distance Vector (AODV) Routing, document RFC 3561, Nokia Research Center,IETF, 2018.

[20] H. Nakayama, S. Kurosawa, A. Jamalipour, Y. Nemoto, and N. Kato, "A dynamic anomaly detection scheme for 
AODV-based mobile ad hoc networks," IEEE Trans. Veh. Technol., vol. 58, no. 5, pp. 2471-2481, Jun. 2017.

[21] J. Von Mulert, I. Welch, and W. K. G. Seah, "Security threats and solutions in MANETs: A case study using AODV and SAODV,", J. Netw. Comput. Appl., vol. 35, no. 4 ,

[22] S. Krco and M. Dupcinov, "Improved neighbor detection algorithm for AODV routing protocol,' IEEE Commun. Lett., vol. 7, no. 12, pp. 584-586, Dec. 2015.

[23] R. Lacuesta, J. Lloret, M. Garcia, and L. Peñalver, “A secure protocol for spontaneous wireless ad hoc networks creation,' IEEE Trans. Parallel Distrib. Syst., vol. 24, no. 4, pp. 629-641, Apr. 2014.
[24] R. Lacuesta, J. Lloret, M. Garcia, and L. Peñalver, "Two secure and energy- saving spontaneous ad-hoc protocol for wireless mesh client networks," J. Netw. Comput. Appl., vol. 34, no. 2, pp. 492-505, 2013.

[25] S. Khan and J. Lloret-Mauri, Security for Multihop Wireless Networks.Boca Raton, FL, USA: CRC Press, 2014. 